\title{
Ein Ausblick zu Nachhaltigkeit und Erneuerungspotenzial an Schweizer Bibliotheken
}

Die vorliegende Festschrift widmet sich, wie im Titel festgehalten, dem Thema ,Innovation durch Kooperation'. Die Beiträge aus verschiedenen Bibliotheken oder Universitäten der Schweiz zeigen die Bandbreite und Vielzahl der kooperativen Vorhaben. Viele Dienstleistungsangebote, die aus solchen Kooperationen hervorgegangen sind, gehören inzwischen fest zum Produktportfolio und Selbstverständnis Schweizer Bibliotheken. Man denke hier beispielsweise an Plattformen für digitalisierte historische Bestände wie e-rara.ch oder E-Periodica, an swissbib, als nationaler Metakatalog, oder an die Kooperative Speicherbibliothek in Büron, Kanton Luzern. Mit der Gründung der Swiss Library Service Platform (SLSP) AG im Mai 2017 wurde sogar eine eigene Governancestruktur für die Zusammenarbeit zwischen den Hochschulbibliotheken ins Leben gerufen. ${ }^{1}$

Der Grossteil dieser Initiativen wurde ermöglicht durch projektgebundene Beiträge im Rahmen des nationalen Förderinstruments der Kooperations- und Innovationsprojekte, das zum Ziel hat, die Hochschulentwicklung auf Bundesebene durch periodische Förderprogramme zu optimieren. ${ }^{2}$

Das Bedürfnis zur stärkeren Kooperation wird sowohl von den Bibliotheken selbst gesucht als auch von der Schweizer Hochschulpolitik unterstützt. An der Zentralbibliothek Zürich wird die Kooperation unter anderem als geeignete Möglichkeit betrachtet, mit den aktuellen Herausforderungen auf technischer und wirtschaftlicher Ebene umzugehen. Dieser Ansatz ist in der Strategie ZB 2020 wie folgt verankert:

Die Digitalisierung verändert und erweitert das Aufgaben- und Dienstleistungsportfolio von
Bibliotheken wie der ZB grundlegend. Dabei eröffnen die modernen Technologien neue
Möglichkeiten für eine effizientere Informationsversorgung. Zugleich zeichnet sich in der
nationalen und internationalen Entwicklung immer deutlicher ab, dass aus Gründen der
Wirtschaftlichkeit und der immer komplexeren technischen Anforderungen stärker koope-
rative Lösungen mit anderen Bibliotheken sowie mit Rechenzentren gesucht werden. Ver-
änderungs- wie Kostendruck sind für die ZB Herausforderung und Chance zugleich, mit

$1 \mathrm{Zu}$ allen genannten Projekten finden sich Beiträge im vorliegenden Band.

2 Vgl. den Beitrag „Nationale Förderprogramme“ von Alice Keller in diesem Band.

Ә Open Access. () Alice Keller, publiziert von De Gruyter. (c) BY-NC-ND Dieses Werk ist lizenziert unter der Creative Commons Attribution-NonCommercial-NoDerivatives 4.0 Lizenz. 
ihren Partnern organisationsübergreifend zeitgemässe Lösungen für kooperative Infrastrukturen und Dienstleistungen zu erarbeiten. ${ }^{3}$

Sinn und Zweck von Kooperationen zwischen Bibliotheken werden vermutlich aus Sicht von Fachkollegen, Politikern und Nutzern kaum je in Frage gestellt. Andererseits ist jedoch das Dreiecksverhältnis von Kooperationen verbunden mit zeitlich befristeten Projekten und dem Anspruch auf Innovation kein einfaches. Das Erfolgspotenzial oder die Praxistauglichkeit dieses Dreiecksverhältnisses soll mittels zweier Fragen analysiert werden. Erstens: Wie nachhaltig sind die durch nationale Förderprogramme lancierten Projekte? Und zweitens: Wie hoch ist das Innovations- oder Erneuerungspotenzial der Schweizer Bibliotheken insbesondere vor dem Hintergrund kooperativer Projekte? Um die Beantwortung dieser Fragen geht es in diesem Ausblick.

\section{Wie nachhaltig sind die durch nationale Förderprogramme lancierten Projekte?}

Über projektgebundene Beiträge des Bundes konnten bisher knapp 60 Bibliotheksprojekte erfolgreich initiiert werden. ${ }^{4}$ Sechs dieser Projekte wurden von der Verfasserin im Rahmen einer Studie auf ihre Nachhaltigkeit hin untersucht und dienen als Basis für die nachfolgenden Ausführungen. ${ }^{5}$ Als nachhaltig wird ein Projekt bezeichnet, wenn es gute Qualität und Wirkung aufweist und über das Projektende hinaus einen dauerhaften Nutzen bietet. Ausserdem ist zur Sicherung der Dauerhaftigkeit die Intervention organisatorisch, strategisch und finanziell in der Trägerorganisation verankert und verfügt ausserdem über das Potenzial, erfolgreich auf andere Kontexte transferiert zu werden. ${ }^{6}$

3 ZB Strategie 2020: Die Zentralbibliothek Zürich zeigt Profil. Zürich 2016, hier S. 8 (https://www. zb.uzh.ch/ueberuns/ZBStrategie/index.html.de). Alle Links in diesem Beitrag wurden am 26.03.2018 überprüft.

4 Vgl. Anhang zum Beitrag „Nationale Förderprogramme“ von Alice Keller in diesem Band.

5 Vgl. hierzu Alice Keller: Nationale Förderprogramme: eine Analyse der Nachhaltigkeit von Bibliotheksprojekten. Bern 2017. In dieser Studie wurden folgende Projekte untersucht: E-Depot, e-rara.ch, Kartenportal.CH, Aufbau Konsortium, swissbib und ElibEval. Die Studie hat nicht den Anspruch, eine umfassende oder repräsentative Aussage zur Nachhaltigkeit von Bibliotheksprojekten allgemein zu machen. Dennoch lassen sich einige generelle Aussagen aus den Resultaten ableiten.

6 Zur Definition der Nachhaltigkeit siehe Alice Keller, Nationale Förderprogramme (wie Anm. 5), S. 7-8. 
Fünf der sechs untersuchten Projekte wurden von den befragten Projektleitern grundsätzlich als nachhaltig bezeichnet. Allerdings bedeutet dies nicht, dass alle Kriterien der Nachhaltigkeit bei allen Projekten erfüllt sind. So werden beispielsweise bei e-rara.ch noch nicht alle digitalisierten Drucke vollständig langzeitarchiviert, obwohl das Projekt in allen anderen Bereichen als nachhaltig bezeichnet werden kann. ${ }^{7}$ Beim Metakatalog swissbib andererseits fehlt ein nachhaltiges Finanzierungsmodell; das Projekt ist weiterhin von Bundesmitteln abhängig. ${ }^{8}$ Das Projekt ElibEval konnte zwar erfolgreich in das Schweizer Kompetenzzentrum für die Evaluation von Online-Angeboten (CHeval) integriert werden, aber auf der Seite der Fachhochschule fehlen die Mittel zur Weiterentwicklung des Angebots. Das Konsortium gilt als ein sehr erfolgreiches Serviceangebot, allerdings werden die Strukturen als nicht ausreichend tragfähig beurteilt. ${ }^{9}$ Einzig das Kartenportal.CH erfüllt alle Kriterien der Nachhaltigkeit. ${ }^{10}$ Das E-Depot, ein konsortialer Server zur zentralen Speicherung lizenzierter Zeitschrifteninhalte, hingegen wurde nach Projektende eingestellt. Dieser Service scheiterte unter anderem daran, dass das System seinerzeit nicht skalierbar war, sowohl aus Sicht der Technik als auch aufgrund der Organisation. Inzwischen stehen für E-Journals auf internationaler Ebene andere Archivlösungen zur Verfügung.

Es haben sich folgende Erfolgsfaktoren herauskristallisiert, die positiv zur Nachhaltigkeit von Projekten beitragen:

- Die gute Zusammenarbeit zwischen den beteiligten Bibliotheken: Die Partner identifizieren sich mit dem Service und tragen ihn ,ideell' mit.

- Gemeinsame Weiterentwicklungen wirken stark, verbindend‘: Die Partner tragen aktiv zur Zukunft des Services bei und übernehmen Verantwortung.

- Die gute Qualität der Services sowohl hinsichtlich der Funktionalitäten als auch der darin enthaltenen Inhalte (Daten oder Digitalisate); sie gelten auch über die Schweizer Grenze hinaus als Referenzmodelle oder State of the Art.

- Die personelle Kontinuität wird durch langjährige, engagierte Projektleiter und Fachspezialisten gewährleistet.

- Open Access: Die Angebote sind für den Endkunden kostenlos.

Fast alle Projekte bzw. Services beruhen in hohem Masse auf Basisleistungen, die von Bibliotheken im Rahmen ihrer Kernaufgaben erbracht werden (Metadatenerfassung bzw. Katalogisierung, Digitalisierung, Lizenzerwerb bei E-Medien). Zu-

7 Vgl. hierzu den Beitrag „E-rara.ch“ von Meda Diana Hotea in diesem Band.

8 Vgl. hierzu den Beitrag „Swissbib“ von Günter Hipler, Nicolas Prongué und René Schneider in diesem Band.

9 Vgl. hierzu den Beitrag „Konsortium“ von Rafael Ball und Pascalia Boutsiouci in diesem Band. 10 Vgl. hierzu den Beitrag „Kartenportal.CH“ von Jost Schmid-Lanter in diesem Band. 
sätzlich profitieren sie von Overhead-Leistungen, die von beteiligten Bibliotheken i.d.R. kostenlos zur Verfügung gestellt werden. Viele Personalkosten laufen ebenfalls über die Trägereinrichtungen. Eine Ausnahme bilden das Konsortium und swissbib, wo die Geschäftsstellen über eigenes Personal (meist in befristeter Anstellung) verfügen. Insgesamt betrachtet, profitieren die beteiligten Bibliotheken davon, dass die Resultate ihrer Kernaufgaben durch die neuen Services optimal der Öffentlichkeit präsentiert und breit genutzt werden. Ausserdem erzielen sie dank Bündelung von Kräften Synergien und eine stärkere Marktposition. Es herrscht also eine ,Win Win-Situation' für das Projekt und die beteiligten Bibliotheken.

Beachtet man die Auflage der Geldgeber, dass Eigenleistungen in der Höhe von mindestens 50\% erbracht werden müssen und es sich ausschliesslich um Anschubfinanzierungen handelt, so überrascht es nicht, dass die Bibliotheken Projekte wählen, die auf ihren Kernaufgaben aufbauen. Schliesslich ist allen klar, dass man nach Ablauf der Projektphase auf eigenen Beinen stehen muss. Dies dürfte einerseits die Nachhaltigkeit positiv beeinflussen, andererseits werden hierdurch Projektvorschläge für radikale Innovationen erschwert oder gar verhindert. Projekte bleiben typischerweise Zusatzdienste, oft sehr wichtige, manchmal auch nur ,Nice to have'-Angebote.

Wichtig ist auch die Anerkennung der Grenzen der Projekte hinsichtlich ihrer Nachhaltigkeit. Obwohl wie eingangs erwähnt viele der neu entstandenen Services und Infrastrukturen nicht mehr wegzudenken sind, ist es bisher keinem der Services gelungen, Projektstellen zu verstetigen bzw. neue, unbefristete Personalstellen zu schaffen. Wie das Beispiel des Konsortiums zeigt, bräuchte es hierfür tragfähigere Strukturen und eine gesicherte dauerhafte Finanzierung. ${ }^{11}$

Dieses Manko wird auch von swissuniversities erkannt und es laufen gegenwärtig intensive Planungen zur Einrichtung einer Koordinationsstelle Wissenschaftliche Information, deren Aufgabe es sein soll, ab 2020 das Servicenetzwerk, das im Rahmen des Programms entsteht, aufzubauen und zu begleiten. ${ }^{12}$ Die Koordinationsstelle soll selbst keine eigenen Services erbringen oder entwickeln; Entwickler und Anbieter der Services bleiben autonom. Der Fokus der Koordinationsstelle liegt vielmehr auf den Bereichen Enabling, Broker, Marketing, Coordi-

11 Gegenwärtig handelt es sich beim Konsortium um eine, einfache Gesellschaft‘. Eine einfache Gesellschaft (eG) ist im schweizerischen Gesellschaftsrecht eine Rechtsform für die Erreichung eines gemeinsamen Ziels. Die Gründung erfolgt durch gegenseitige übereinstimmende Willensäusserung.

12 Vgl. hierzu „Wissenschaftliche Information: Projekt ,nationale Organisation““: https://www. swissuniversities.ch/de/organisation/projekte-und-programme/p-5/projekt-nationale-organisati on. 
nation, Label und Support, ${ }^{13}$ wobei sich der Bereich Enabling auf eine Anschubfinanzierung und der Support auf die Unterstützung in Rechtsfragen und bei der Rechnungsstellung beschränken. Die Verantwortung für die Aufrechterhaltung der neuen kooperativen Services wird also auch weiterhin bei den Bibliotheken liegen. Diese sind nun aufgefordert, selbst Strategien $\mathrm{zu}$ entwickeln, wie die neuen Services mit eigenen Mitteln langfristig aufrechterhalten werden können.

Ein Blick auf die Kosten dieser gemeinsamen Services für die eigene Bibliothek zeigt, dass sie im Einzelnen nicht ausserordentlich hoch sind. Schliesslich war man sich von Anfang an klar, dass die Ressourcen knapp waren. Dennoch kumulieren sich die Kosten, wenn die Bibliotheken an diversen Projekten beteiligt sind, und es ist erfahrungsgemäss schwierig, diese zusätzlichen Kosten oder Stellenprozente in das Regelbudget aufzunehmen. Hier besteht aus Sicht mancher Fachkollegen Handlungsbedarf auf der Ebene der nationalen Gremien und bei swissuniversities.

\section{Erneuerungspotenzial der Schweizer Bibliotheken vor dem Hintergrund kooperativer Projekte}

Während es in der ersten Frage um die Nachhaltigkeit von Projekten ging, steht bei der zweiten Frage das Erneuerungspotenzial des im Untertitel dieses Buchs aufgezeigten Modells Innovation durch Kooperation im Fokus.

Ein Blick in die Fachliteratur zeigt, dass die Begriffe ,Nachhaltigkeit' und ,Innovation“ auch als Gegensatzpaar gesehen werden können. Diesem Gedanken zufolge bezieht sich die Nachhaltigkeit auf Etabliertes und Bewährtes, Stetigkeit, die Fortführung von Bewährtem und Sicherheit. Im Gegensatz dazu steht Innovation für etwas Neuartiges, für Veränderungen, Risiko und geringe Planbarkeit, Komplexität und für Konfliktpotenzial unter den Involvierten. ${ }^{14}$

In der Tat bestätigen Gespräche mit Fachkollegen, dass die Kombination von hohem Eigenanteil, relativ kurzen Projektlaufzeiten, überschaubaren Fördersummen und Forderung nach Nachhaltigkeit grosse Sprünge und hiermit auch grundlegende Innovationen verhindern. Bei den meisten nationalen Projekten handelt es sich um Zusatzservices, aufbauend auf dem Kerngeschäft der Bibliothek, nicht um radikale Neuerungen. Es sind Kooperationsprojekte, die vor allem die Bünde-

13 Gemäss Entscheidungsgrundlage zur Koordinationsstelle „Wissenschaftliche Information“ vom 12.09.2017 (internes Dokument).

14 Sabine Seufert, Dieter Euler: Nachhaltigkeit von eLearning-Innovationen. Ergebnisse einer Delphi-Studie. St. Gallen 2004 (SCIL-Arbeitsbericht 2). 
lung von Kräften zur Maximierung der Sichtbarkeit und damit auch der Nutzung von Beständen fördern. Erneuerungen radikaler Art, die es den Schweizer Bibliotheken beispielsweise ermöglichen würden, in Konkurrenz zu Internetunternehmen, multinational agierenden Verlagskonzernen oder Big Data-Grossprojekten $\mathrm{zu}$ treten, fehlen. Aber vielleicht ist das auch zu viel verlangt von einem kleinen Land wie der Schweiz!

Blickt man über die Landesgrenze auf die deutsche Fachliteratur, so erkennt man ein klares Plädoyer für tiefgreifende Veränderungen, die deutlich über das Mass von kooperativen Projekten zur Serviceoptimierung hinausgehen. Andreas Brandtner, UB Mainz, spricht von einer „scheinbar paradoxen Situation“15: Auf der einen Seite agiert die bibliothekarische Gemeinschaft konform mit den Grundwerten der Informations-, Wissens- bzw. Mediengesellschaft. Auf der anderen Seite begünstigen die Entwicklungen hin zu diesen neuen Gesellschaftsformen keineswegs den Organisationstyp Bibliothek. Die Bibliotheken, die bisher von ihrer Quasi-Monopol-Position als Informationsspeicher und -versorger profitierten, befinden sich laut Brandtner nun „in einer Marktsituation, die sich durch disruptive Innovation, harte Konkurrenz und hochgradige Rasanz auszeichnet.“ (ebd.) Elmar Mittler bezieht sich in seinem Aufsatz Wohin geht die Reise? u.a. auf Brandtner und fordert zur Bewältigung dieser aktuellen Herausforderungen grundlegende strukturelle Organisationsänderungen an den Bibliotheken. Er stellt fest, dass mancher Leitende „vor dem Aufwand und dem in Umsetzung auch für die Mitarbeiter teilweise schmerzhaften und deshalb schwer durchsetzbaren Prozess“ zurückschrecken mag (ebd.). ${ }^{16}$ Es fehle oft an „Mut und Entschlossenheit“ auf Leitungsebene. Auch Wilfried Sühl-Strohmenger erwähnt dieses Manko in seiner Rezension zum Praxishandbuch Bibliotheksmanagement, ${ }^{17}$ einem Werk, das den Anspruch hat, „einen Überblick über alle Aspekte des Bibliotheksmanagements in Deutschland“ zu bieten. ${ }^{18}$ Laut Sühl-Strohmenger fehlt es in Deutschland an übergreifenden Konzepten für ein neu zu strukturierendes Management in Wissenschaftlichen Bibliotheken, das den Herausforderungen des

15 Andreas Brandtner: Wandel - Krise - Transformation. In: Rafael Ball, Stefan Wiederkehr (Hrsg.): Vernetztes Wissen. Online. Die Bibliothek als Managementaufgabe. Festschrift für Wolfram Neubauer zum 65. Geburtstag. Berlin/Boston 2015, S. 63-75, hier S. 64.

16 Elmar Mittler: Wohin geht die Reise? Bibliothekspolitik am Anfang des 21. Jahrhunderts. In: Bibliothek Forschung und Praxis 41/2 (2017), S. 213-223, hier S. 221.

17 Wilfried Sühl-Strohmenger: Rezension zu: Rolf Griebel, Hildegard Schäffler und Konstanze Söllner (Hrsg.): Praxishandbuch Bibliotheksmanagement. Berlin u.a. 2015. In: o-bib 2/1 (2015), S. 47-55, hier S. 54.

18 Siehe Produktinfo zum Praxishandbuch Bibliotheksmanagement auf der Verlagshomepage htt ps://www.degruyter.com/view/product/203487. 
digitalen Zeitalters und den damit einhergehenden Veränderungen der Informations- und Wissenschaftspraxis entspricht.

In der Schweiz ist man sich unter Fachkollegen dieser Notwendigkeit der Veränderung durchaus bewusst. Die Diskussion ,Wohin geht die Reise?` wird in der Schweiz allerdings stark auf der fachlich-inhaltlichen Seite geführt, kaum auf der Seite der Organisationsentwicklung. Es herrscht aus Sicht der Verfasserin eine starke Verteidigungshaltung gegenüber den Werten und den Aufgabenverteilungen einer traditionellen (aber modernen) Bibliothek. So hat ein Versuch von Rafael Ball, die Rolle der Bibliotheken grundsätzlich in Frage zu stellen, schärfste Kritik sowohl von Bibliothekaren als auch von Bibliotheksbenutzern geerntet. ${ }^{19}$ Die Schweizer Bibliothekare sind stolz auf ihre Bibliotheken, Sammlungen und Errungenschaften der letzten Jahre und distanzieren sich bewusst von dieser Art der „Disruption“. 20

Aus Sicht der Verfasserin haben die Wissenschaftlichen Bibliotheken der Schweiz in mancher Hinsicht klaren Aufholbedarf. Services zur Publikations- und Forschungsunterstützung, zur Förderung von Open Access oder Forschungsdatenmanagement sind weniger weit fortgeschritten als in anderen europäischen Ländern. Im Vergleich zu den angelsächsischen Ländern, wo bereits in den 80er und 90er Jahren eine zunehmende Hinwendung zur benutzerorientierten Bibliotheksarbeit vollzogen worden ist, arbeiten die Schweizer Bibliotheken weiterhin stark bestandsorientiert. Als Vorteil dieser Bestandsorientierung können die Benutzenden an fast allen Wissenschaftlichen Bibliotheken der Schweiz auf ausgezeichnete Sammlungen sowohl in gedruckter als auch in elektronischer Form zugreifen. Darüber hinaus haben sie über leistungsfähige Dokumentliefermodelle schnellen Zugang zu Beständen anderer Bibliotheken. Die Speicherbibliothek im luzernischen Büron ist ein äusserst erfolgreiches Beispiel einer modernen kooperativen Printarchivlösung.

Der Nachteil dieser starken Fokussierung auf den Bestand, wie sie in der Schweiz praktiziert wird, liegt darin, dass diese klassischen Bibliotheksbereiche als wenig ,trendig، oder zukunftsweisend gelten, sehr personalintensiv sind und entsprechend wenig freie Kapazitäten für neu aufkommende Arbeitsfelder zur Verfügung stehen. Eigene Erfahrungen zeigen auch, dass für die meisten Mitarbeitenden in Bibliotheken der konkrete Nutzen dieser ,neuen Services' noch zu wenig greifbar ist. Sie möchten verständlicherweise die über Jahre erreichten

19 Michael Furger: „Bibliotheken: Weg damit!“ Interview mit Rafael Ball. In: NZZ am Sonntag, 07.02.2016 (https://nzzas.nzz.ch/hintergrund/bibliotheken-und-buecher-weg-damit-meint-rafael-ball-ld.147683). Siehe auch die Leserkommentare in der NZZ am Sonntag vom 14.02.2016.

20 Rafael Ball: Digitale Disruption. Warum sich Bibliotheken neu positionieren müssen. In: Forschung \& Lehre 23/9 (2016), S. 776-777. 
hohen Servicelevels und Qualitätsniveaus nicht ohne für sie einsichtigen Grund aufgeben. Vor dem Hintergrund, dass in den meisten Schweizer Bibliotheken kein grosser Kostendruck spürbar ist, der eine grundsätzliche Umverteilung bzw. Kürzung der Ressourcen notwendig macht, sind Abstriche in diesen klassischen Bibliotheksbereichen auch gegenüber den Kunden schwierig zu argumentieren.

Die erforderliche Freispielung von Fachkräften für neue Aufgaben soll nach Ansicht vieler Fachkolleginnen und -kollegen über die Umsetzung der Swiss Library Service Platform angegangen werden. So heisst es im Projektantrag:

Der Bibliothekskunde steht mit seinen Anforderungen im Zentrum, und diese können besser bedient werden, wenn sich Bibliotheken in der Schweiz auf einer nationalen Ebene zusammenschliessen und Massen- und Routinearbeiten, wo dies möglich ist, zentral in einem Service-Netzwerk bündeln und die Ergebnisse an einer Stelle zur Verfügung stellen. Die dadurch in den einzelnen Bibliotheken frei werdenden Ressourcen können eingesetzt werden, um individuell und unmittelbar auf die Wünsche und Bedürfnisse der Kunden an den jeweiligen Standorten einzugehen. ${ }^{21}$

Wie diese freigespielten Kapazitäten in Zukunft eingesetzt werden sollen, ist nicht Teil des Projektes SLSP, sondern liegt im Ermessensspielraum der einzelnen Bibliotheken. Insgesamt kann man davon ausgehen, dass die Stärkung von Services zur Forschungsunterstützung weit oben auf der Agenda der Bibliotheken stehen wird. Einerseits scheint es noch verfrüht im Detail zu planen, schliesslich ist der Go-Live-Termin der neuen Plattform erst für 2020/2021 geplant, andererseits möchten die Mitarbeitenden bereits heute mehr über ihre zukünftigen Arbeitsfelder wissen und sich im Rahmen von Weiterbildungen oder persönlichen Vertiefungen gezielt vorbereiten können. Vor diesem Dilemma ist es für die Leitungsebene nicht immer einfach, den richtigen Moment für die Aufgleisung eines Changeprozesses zu finden.

Eine grosse strukturelle Veränderung, die mit SLSP zusammenhängt, ist allerdings bereits heute spürbar und sie wird von den beteiligten Fachspezialisten auch positiv aufgenommen. Bisher orientierten sich die Entwicklungen an Schweizer Bibliotheken stark an den meist regional ausgerichteten Verbünden. In Zukunft wird ein Umdenken hin zu nationalen Standards und Services notwendig sein, verbunden mit der besonderen Herausforderung der Mehrsprachigkeit. Die Arbeit in den heutigen SLSP-Gremien zeigt, dass der Aufbau dieses Servicenetzwerks und die Zusammenführung von Daten aus verschiedenen Bibliothekskatalogen ausgesprochen zeit- und ressourcenintensiv ist, so dass die oben erwähnte

21 Kurzversion des SLSP Projektantrags siehe https://blogs.ethz.ch/slsp/2015/10/28/francais-de r-projektantrag-slsp-zum-downloaden/, hier S. 4. 
Vorstellung von freien Kapazitäten noch in weiter Zukunft scheint! Dennoch sind sich vermutlich alle Schweizer Bibliothekarinnen und Bibliothekare einig, dass die Erneuerung des Schweizer Bibliothekswesens nur über diesen Weg - nämlich über die Umsetzung von SLSP als gemeinsamer Serviceplattform - erfolgen kann. In diesem Sinne ist der Untertitel des Buches Innovation durch Kooperation auch Programm für das Bibliothekswesen der Schweiz. 
